\title{
Somogy megye lágytestú és felemás lábfejízes bogarainak katalógusa (Coleoptera: Elateroidea (részben), Lymexyloidea, Cleroidea, Tenebrionoidea)
}

\author{
SZALÓKI DEZSÖ
}

\begin{abstract}
SZALÓKı D.: Catalogue of the Malacodermata and Heteromera of Somogy county (Coleoptera: Elateroidea (in part), Lymexyloidea, Cleroidea, Tenebrionoidea)

Abstract: Now 316 species (59 Elateroidea (in part), I Lymexyloidea, 68 Cleroidea, 188 Tencbrionoidea) are known in the fauna of Somogy county; it is $53 \%$ of Hungarian fauna. The first occurrence of Pytho depressus (Linnaeus, 1767) in Hungary is reported from this region.
\end{abstract}

\section{Bevezetés}

Az elsó Somogyra vonatkozó adatokat POLINSzKy (1886) közli. Cikkében összesen 13 Lampyridae, Ciidae, Tenebrionidae és Meloidae faj szerepel. Az itt szerepló Corticeus ametarius H. ma érvényes nevét egyetlen katalógusból sem sikerült megállapítani. A Fauna Regni Hungariae (KUTHY 1990) 7 faj siófoki lelóhelyét közli. Ez rendkívül kevés adat, de akkoriban a terepi kutatás Budapest környékére, a Kárpátokra és a Tengermellékre korlátozódott.

A következó adatokat a Magyarország Állatvilága KASZAB által írt füzeteiben találjuk. Munkáiban (1955) 18, (1956) 10, (1957) 8 és (1979) 18 fajt említ, zömében siófoki és balatonöszödi lelóhellyel. A megírás során feldolgozta a Természettudományi Múzeum anyagát, az adatokat részletesen HoRVN'(OVICH (1969) és TótH (1973, 1981) közli. Horvatovich a Malacodermata hadba tartozó családok (Lycidae, Lampyridae, Drilidae, Cantharidae, Malachiidae, Dasytidae, Cleridae, Corynetidae és Lymexylonidae) 1955 után a Természettudományi Múzeumba került példányait is meghatározva, 122 faj adatát közli. Tóth a Heteromera hadba tartozó családokkal foglalkozik, a Természettudományi Múzeum anyagán kívül a veszprémi Bakony Múzeum és saját gyújteményének adatait is közli. 1973-ban a Meloidae család 28 fajának, 1981 ben az Anthicidae, Aderidae, Scraptiidae, Pyrochroidae, Pythidae és Oedemeridae családok 48 fajának adatait közli. Az adatok többsége Siófokról, Zamárdi: Töreki-lápról, Balatonöszödröl, Kaposvárról és a Kis-Balatonról származik. TótH (1981) tévesen említi az Oedemeridae családban az Ischnomera caerulea (Linnaeus, 1758) fajt. Átnézve az Természettudományi Múzeum anyagában található somogyi példányokat, valamennyi az Ischnomera cyanea (Fabricius, 1787) faj példányainak bizonyultak. A tévedést az okozta, hogy KAszıß (1956) a cyanea-t szinonimnak tekintette. A korábban határozott példányokon még mind a két név szerepel. LOHSE \& LUCH'T (1992) közli a két faj hímjének paramera- és penisvég rajzait, mely alapján a két faj jól elkülönül. 
1975-tôl a Barcsi Borókás TK. - a Janus Pannonius Múzeum (Pécs) által szervezett - kutatása volt az első komplex jellegú fauna feltárást célzó vizsgálat Somogy megyében. Ennek keretében Horvatovich (1976, 1979, 1980, 1981.) négy rövid cikkben II faj adatát közli.

A Magyarország Állatvilága sorozatban MERKL (1993) l fajt, MERKL \& SLiPINSZKI (1993) 9 fajt említ Somogyból. SzAlóKI (1997) a Mátra Múzeum (Gyöngyös) gyúiteményéból 4 faj adatait közli. A Dunántúli Dolgozatok Duna-Dráva Nemzeti Park faunájával foglalkozó kötetében MerkL (1989) 68, SZALóKi (1998) 3 l fajt említ a Dráva mente Somogyhoz tartozó területeiról.

A Pytho depressus (Linnaeus, 1767) legkorábbi magyarországi eloofordulása: Pusztakovácsi, Alsókölked, 1997.X.18. leg.: Rozner Gy. (Sর́r et al. 2001).

A Balaton déli partja a utóbbi évtizedekben gyökeresen megváltozott. A területeket jobbára beépítették, lecsapolták, azért Siófok, Zamárdi és Balatonöszöd környékének hajdan gazdag faunája mára átalakult, feltehetôen erôsen degradálódott. A valaha kiterjedt sztyepterületek zsugorodása miatt a Meloidae család faj és egyedszáma csökkent a legjobban.

A családok családsorozatba osztásánál LAWRENCE \& NEwTON (1995) és SLIJINSKI \& LAWRENCE (1999) munkáit vettem alapul:

Elateroidea: Cerophytidae, Eucnemidae, Throscidae, Elateridae, Drilidae, Omalisidae, Lycidae, Lampyridae, Cantharidae

Lymexyloidea: Lymxylidae

Cleroidea: Phloiophilidae, Trogossitidae, Cleridae, Melyridae

Tenebrionoidea: Mycetophagidae, Ciidae, Tetratomidae, Melandryidae, Mordellidae, Rhipiphoridae, Zopheridae, Tenebrionidae, Prostomidae, Oedemeridae, Meloidae, Mycteridae, Pythidae, Pyrochroidae, Salpingidae, Anthicidae, Aderidae, Scraptiidae.

A Phloiophilidae, Tetratomidae, Rhipiphoridae és Prostomidae család fajai nem ismertek Somogy megyéból.

A régebben külön családsorozatba tartozó Cerophytidae, Eucnemidae, Throscidae, Elateridae családokkal nem foglalkozok cikkemben.

A nevezéktanban LOHSE \& LUCHT (1992) munkáját vettem alapul. A felhasznált forrásokban közölt nevek egy része eltér attól, amelyet ma érvényesnek tekintenek; ilyen esetekben az eredetileg használt név zárójelben egyenlőségjel után olvasható.

\section{Somogyból ismert fajok jegyzéke}

\section{Clerroiden}

Drilidae

Dithes concolor-Nhrens, 1812 - Horvatorich 1969

\section{Omalisidae}

Omalisus fontisbellaquaei Fourcroy, 1785 (=Homalisus fontisbellaquaei) - Horvatovich 1969, Szalóki 1998
Lycidae Pyropterus nigronuber (De Gicer, 1774) Szalóki 1998 Dictyopterus aurora (Herbst, 1784) . Horvatovich 1981, Szalóki 1998

Lygistopterns sanguineus (Linnacus, 1758) Horvatovich 1969, 1981, Šalóki 1998

Lampyridae Lampyris noctiluca (Linnacus, 1767) - Polinszky 


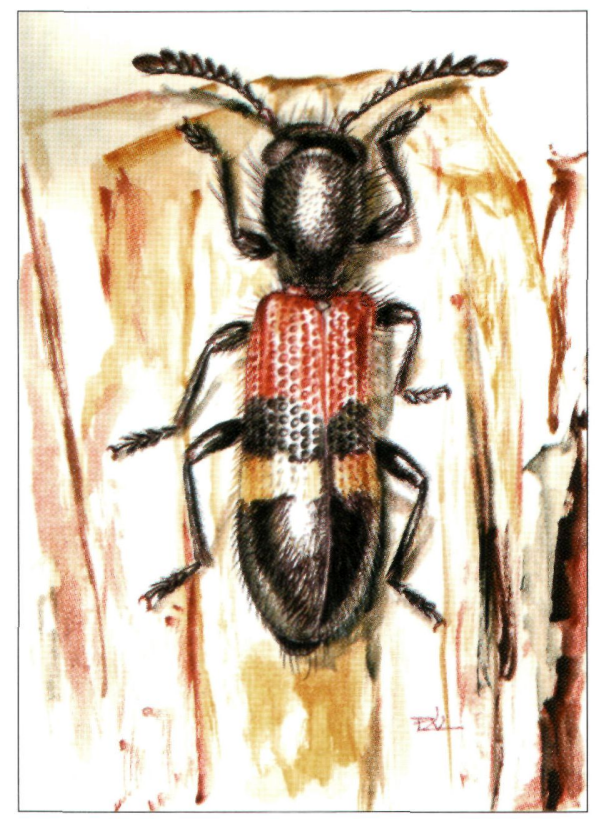

1. ábra: Egysávos szúfarkas (Tilloidea unifasciata - Cleridae)

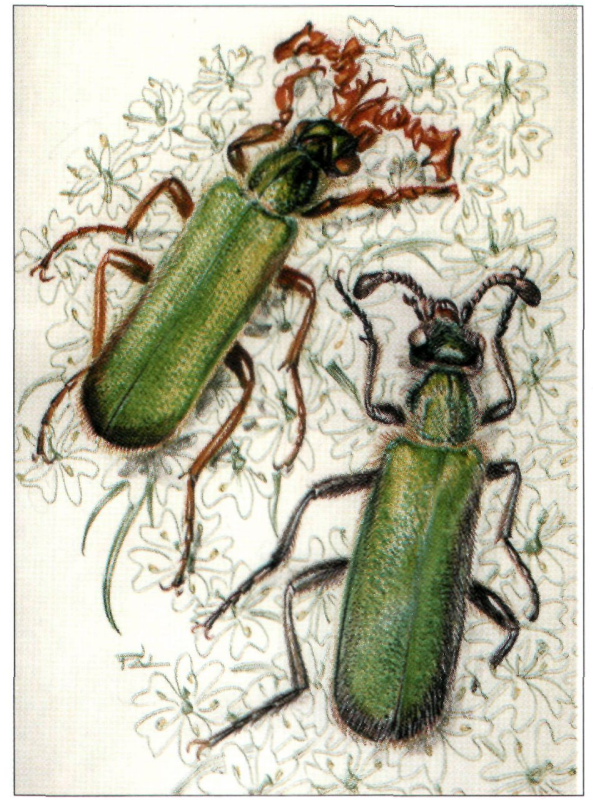

2. ábra: nagy torzcsápúbogár (Ceroscoma scheberi - Meloidae) 


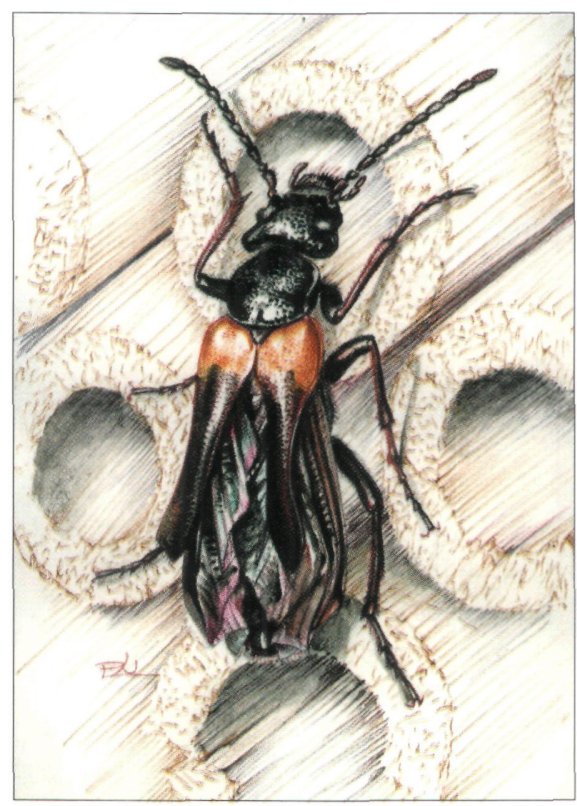

3. ábra: Piroslábú méhbogár (Sitaris muralis - Meloidae)

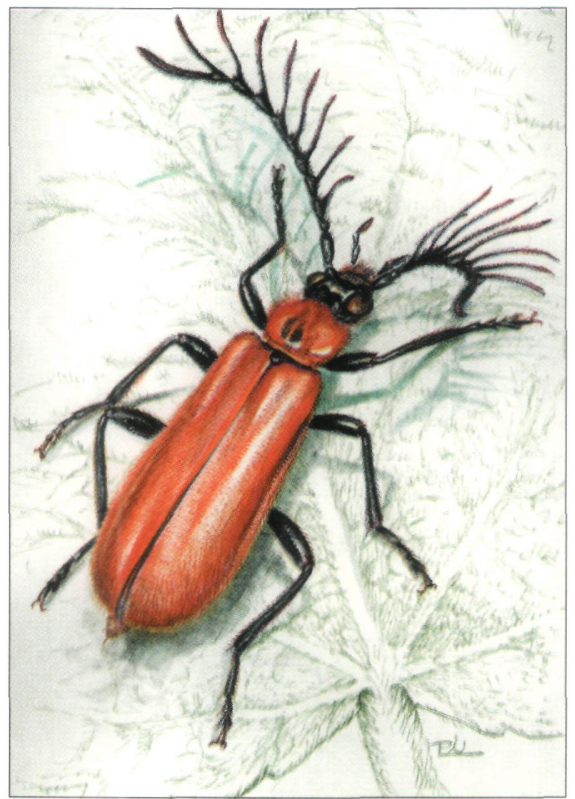

4. ábra: Kis bíborbogár (Schizotus pectinicornis - Pyrochroidae) 
1886, Horvatovich 1969, S\%alöki 1998

Lamprohiza splendidula (Linnacus, 1767) -

Horvatovich 1969

Phosphaenus hemipterus (Gicoffroy, 1762) -

Horvatovich 1969

\section{Cantharidae}

Ancistronycha abdominalis (Fabricius, 1798)

(=Cantharis abdominalis) - Horvatovich 1969

Ancistronycha erichsoni (Bach, 1852)

(=Cantharis erichsoni) - Kaszab 1955 ,

Horvatovich 1969

Cantharis assimilis Paykull, 1798 .

Horvatovich 1969

Cantharis bicolor Herbst, 1784 .

Horvatovich 1969, 1981, Szalóki 1998

Cantharis figurata Mannerhcim, 1843 - Ka-

szab 1955, Horvatovich 1969

Cantharis fulvicollis Fabricius, 1792 .

Horvatovich 1969

Cantharis fusca Linnaeus, 1758 .

Horvatovich 1969, Szalóki 1997, 1998

Cantharis lateralis Linnaeus, 1758 .

Horvatovich 1969, Szalóki 1997

Cantharis liburnica Depoli, 1912 .

Horvatovich 1969

Cantharis livida Linnaeus, 1758 .

Horvatovich 1969, Szalóki 1998

Contharis longicollis (Kiesenwetter, 1859) -

Coll: Szalóki

Cantharis nigricans Müller, 1776.

Horvatovich 1969, Szalóki 1998

Cantharis pagana Rosenhauer, 1847 .

Horvatovich 1969

Cantharis paradoxa Hicker, 1960

Horvatovich 1969

Cantharis pallida Goeze, 1777

Horvatovich 1969, Szalóki 1998

Cantharis pellucida Fabricius, 1792 .

Horvatovich 1969

Cantharis pulicaria Fabricius, 1781

Horvatovich 1969

Cantharis rufa Linnacus, 1758

Horvatovich 1969, Szalóki 1998

Cantharis rustica Fallén, 1807 - Horvatovich 1969

Cantharis sudetica Letzner, 1847 .

Horvatovich 1969

Metacantharis clypeata (Illiger, 1798)

(=Metacantharishaemorthoidalis (Fabricius,

1792)) - Horvatovich 1969

Metacantharis discoidea (Ahrens, 1812) -

Horvatovich 1969

Rhagonycha atra (Linnaeus, 1767)

Horvatovich 1969
Rhagonycha femoralis (Brullé, 1832) -

Horvatovich 1969

Rhagonycha fulva (Scopoli, 1763) -

Horvatovich 1969, Szalóki 1997, 1998

Rhagonycha gallica Pic, 1923 - Kaszal), 1955 .

Horvatovich 1969)

Rhagonycha lignosa (Müller, 1764).

Horvatovich 1969

Rhagonycha limbata Thomson, 1864 -

Horvatovich 1969, Szalóki 1998

Rhagonycha lutea (Müller, 1764).

Horvatovich 1969

Rhagonycha nigriceps Waltl, 1838.

Horvatovich 1969

Rhagonycha rorida Kiesenwetter, 1867 .

Horvatovich 1969

Rhagonycha testacea (Linnaeus, 1758).

Horvatovich 1969, 1981, Szalóki 1998

Cratosilis denticollis (Schummel, 1844) - Ka-

szab 1955, Horvatovich 1969

Silis nitidula (Fabricius, 1792).

Horvatovich 1969

Silis ruficollis (Fabricius, 1775) .

Horvatovich 1969, Szalóki 1998

Malthinus balteatus Suffrian, I85I - Kaszab

1955, Horvatovich 1969

Malthinus fasciatus (Olivier, 1790) -

Horvatovich 1969

Malthinus frontalis (Marsham, 1802) - Kaszab 1955, Horvatovich 1969

Malthinus glabellus Kiesenwetter, 1852.

Horvatovich 1969

Malthinus punctatus (Fourcroy, 1785)

(=Malthinus flaveolus (Herbst, 1786)) -

Horvatovich 1969, Szalóki 1998

Malthinus seriepunctatus Kiesenwetter, 1851 -

Horvatovich 1969

Malchinus demissus Kiesenwetter, 1863 - Kaszab 1955, Horvatovich 1969

Malchinus nigrinus Schaufuss, 1866 .

Horvatovich 1969

Malthodes debilis Kiesenwetter, 1852 -

Horvatovich 1969

Malthodes dieneri Kaszab, 1955 - Kaszab

1955, Horvatovich 1969

Molthodes dimidiaticolis (Rosenhauer, 1847) -

Horvatovich 1969

Malthodes guttifer Kiesenwetter, 1852 .

Horvatovich 1969

Malthodes holdhausi Kaszab, 1955.

Horvatovich 1969

Malthodes marginatus (Latreille, 1806) -

Horvatovich 1969, Szalóki 1998

Malthodes minimus (Linnacus, 1758).

Horvatovich 1969 
Malthodes mysticus Kiesenwetter, 1852. Horvatovich 1969

\section{LYMEXYLOIDEA}

\section{Lymexylidae}

Hylecoetus dermestoides (Limnacus, 1761). Horvatovich 1969

\section{Trogossitidae}

Tenebroides fuscus (Goeze, 1777) - Coll: Szalóki

Grynocharis oblonga (Linnaeus, 1758) Merkl 1993

\section{Cleridae}

Tillus pallidipennis Bielz, 1850 - Kaszab 1955, Horvalovich 1969

Tilloidea unifasciata (Fabricius, 1787) (=Tillus unifasciatus) - Horvatovich 1969

Tarsostenus univittatus (Rossi, 1792) - Kaszab 1955, Horvatovich 1969

Opilo pallidus (Olivier, 1795) - Horvatovich 1969

Clerus mutillarius Fabricius, 1775 (=Pseudoclerops mutillarius)- Horvatovich 1969

Thanasimus formicarius (Linnaeus, 1758) . Horvalovich 1969

Thanasimus rufipes (Brahm, 1797) . Horvatovich 1969

Trichodes alvearius (Fabricius, 1792) - Kas\%ab 1955, Horvatovich 1969

Trichodes apiarius (Linnaeus, 1758). Horvatovich 1969, Szalóki 1998

Trichodes favarius Illiger, 1802 - Horvatovich 1969, Szalóki 1998

Korynetes caeruleus (De Geer, 1775) (=Corynetes obenbergeri (Jannsen, 1936)) - Coll: Szalóki

Korjnetes ruficornis Sturm, 1837 . Horvatovich 1969, Szalóki 1998

Necrobia ruficollis (Fabricius, 1775). Horvatovich 1969

Necrobia rufipes (De Gecr, 1775) Horvatovich 1969

Necrobia violacea (Linnacus, 1758). Horvatovich 1969, Szalóki 1998

Opetiopalpus scutellaris (Panzer, I797) . Horvatovich 1969

\section{Melyridae}

Enicopus hirtus (Linnacus, 1767) (=Henicopus hirtus) - Horvatovich 1969

Aplocnemus impressus (Marsham, 1802 ) (=Haplocuemus impressus)- Horvatovich 1969

Aplocnemus kiesenwetteri Schilsky, 1897 (=Haplocuemus kiesenwetteri)Horvatovich 1969
Aplocnemus nigricornis (Fabricius, 1792) -

(=Haplocuemus nigricomis) Horvatovich 1969

Aplocnemus piertli Schilsky, 1897

(=Haplocuemus piertli) Horvatovich 1969

Trichoceble floralis (Olivier, 1790) (=Julistus

floralis) - Horvatovich 1969

Dasytes aerosus Kiesenwetter, 1867.

Horvatovich 1969, S\%alóki 1998

Dasytes caeruleus (De Geer, 1771) .

Horvatovich 1969

Dasytes fusculus (Illiger, 1801) -

Horvatovich 1969, Sralóki 1998

Dasytes niger (Limnacus, 176I) -

Horvatovich 1969

Dasytes obscumus Gyllenhal, 1813- Kasyab 1955, Horvatovich 1969

Dasytes oertzeni Schilsky, 1895 - Kašab 1955, Horvatovich 1969

Dasytes plumbeus (Müller, 1776).

Horvatovich 1969, Szalóki 1998

Dasytes subaeneus Schönherr, 1817. Horvatovich 1969

Dasytes subalpinus Baudi, 1873 - Kas\%ah 1955, Horvatovich 1969

Dolichosoma lineare (Rossi, 1792)

Horvatovich 1969, Szalóki 1998

Danacea marginata (Küstcr, 1851)

Horvatovich 1969

Danacea morosa Kiesenweller, 1863.

Horvatovich 1969

Danacea nigritarsis (Küster, 1850)

Horvatovich 1969

Danacea pallidipalpis Abeille de Perrin, 1894 Kaszab 1955, Horvatovich 1969

Danacea pallipes (Panzer, 1793).

Horvatovich 1969

Danacea serbica Kiesenwetter, 1863.

Horvatovich 1969

Troglops albicans (Limnaeus, 1767) .

Horvatovich 1969

Troglops cephalotes (Olivicr, 1790) - Kas\%ab

1955, Horvatovich 1969

Hypebaeus flavipes (Fabricius, 1787)

Horvatovich 1969

Charopus concolor (Fabricius, 1801) -

Horvatovich 1969

Charopus flapipes (Paykull, 1798) -

Horvatovich 1969

Charopus thoracicus Morawitz, 1861 -

Horvatovich 1969

Ebaeus appendiculatus Erichson, 1840.

Horvatovich 1969

Ebaeus ater Kiescnwetter, 1863.

Horvatovich 1969 
Ebaeus flavicornis Erichson, 1840 - Ciidae

Horvatovich 1969

Ebaeus gibbus (Drapie\%, 1819) - Kaszab

1955, Horvatovich 1969

Ebaeus pedicularis (Fabricius, 1777) -

Horvatovich 1969

Sphinginus coarctatus (Erichson, 1840) -

Horvatovich 1969

Attalus analis (Panzer, 1796) - Horvatovich 1969

Axinotarsis marginalis (Laporte de Castelnau, 1840) - Horvatovich 1969, Szalóki 1998

Axinotarsis pulicarius (Fabricius, 1775) -

Horvatovich 1969

Axinotarsis ruficollis (Olivier, 1790) -

Horvatovich 1969, Szalóki 1998

Malachius aeneus (Linnaeus, 1758) -

Horvatovich 1969

Malachius bipustulatus (Linnaeus, 1758) -

Horvatovich 1969, Szalóki 1998

Clanoptilus ambiguus (Peyron, 1877) (=Malachius)- Horvatovich 1969

Clanoptilus elegans (Olivier, 1790) (=Malachius elegans)- Kaszab 1955, Horvatovich 1969

Clanoptilus geniculatus (Germar, 1824) (=Malachius geniculatus)- Horvatovich 1969, Szalóki 1998

Clanoptilus marginellus (Olivier, 1790) (=Malachius marginellus)- Horvatovich 1969, Szalóki 1998

Clanoptilus marginellus (Abeille de Perrin, 1885) (=Malachius marginellus)- Horvatovich 1969

Cordylepherus piridis (Fabricius, 1787) (=Malachius viridis)- Horvatovich 1969

Anthocomus bipunctatus (Harrer, 1784) -

Horvatovich 1969

Anthocomus coccineus (Schaller, 1783).

Horvatovich 1969

Cerapheles terminatus (Ménetriés, 1832) -

Horvatovich 1969

Apalochrus femoralis Erichson, 1840 -

Horvatovich 1969, 1981, Szalóki 1998

\section{TENEBRIONOIDEA}

\section{Mycetophagidae}

Mycetophagus decempunctatus Fabricius, 1801 Merkl \& Slipinski 1993

Mycetophagus populi Fabricius, 1798 - Merkl \& Slipinski 1993

Mycetophagus quadripustulatus (Linnaeus, 1761) - Merkl 1998

Typhaea stercorea (Linnacus, 1758) - Merkl 1998

Litargus connexus (Fourcroy, 1785) - Coll: Szalóki

Ennearthron cornutum (Gyllenhal, 1827) Merkl 1998

Cis boleti (Scopoli, 1763) - Merkl 1998

Cis jacquemarti Mellie, 1848 - Polinszky 1886

Cis micans (Fabricius, 1792) - Merk1 1998

Cis nitidus (Fabricius, 1792) - Polinszky 1886, Kuthy 1900

Cis pseudolinearis Lohse, 1964 - Kuthy (1900) Cis perrisi Abeille de Perrin, 1874 néven említi, ez a faj azonban Dél-Franciaországban él.

Orthocis alni (Gyllenhal, 1813) (=Cis alni)Polinszky 1886, Merkl 1998

Orthocis pygmaeus (Marsham, 1802) (=Cis pygmaeus) - Polinszky 1886

Rhopalodontus perforatus (Gyllenhal, 1827) Polinszky 1886

\section{Melandryidae}

Orchesia blandula Brancsik, 1874 - Kaszab 1957

Orchesia fasciata (Illiger, 1798) - Coll: Szalóki

Orchesia micans (Panzer, 1794) Horvatovich 1981, Merkl 1998

Orchesia minor Walker, 1837 - Kaszab 1957

Orchesia undulata Kraatz, 1853 - Merkl 1998

Xylita laerigata (Hellen, 1786) - Coll: Szalóki

Conopalpus testaceus (Olivier, 1790) - Merkl 1998 1998

Osphya bipunctata (Fabricius, 1775) - Merkl

\section{Mordellidae}

Mordella aculeata Liannaeus, 1758 - Kaszab 1979

Mordella purpurascens Apfelbeck, 1914 - Kaszab 1979

Mordella velutina Emery, 1876 - Kaszab 1979

Mordella viridescens Costa, 1854 - Kaszab 1979

Curtimorda bisignata (Redtenbacher, 1849) -

Kaszab 1979

Curtimorda maculosa (Naezen, 1794) - Kaszab 1979

Mordellistena aequalica Ermisch, 1977 - Kaszab 1979

Mordellistena brunneispinosa Ermisch, 1963 . Kaszab 1979

Mordellistena humeralis (Linnacus, 1758) Kaszab 1979

Mordellistena klapperichi Ermisch, 1956 - Kaszab 1979

Mordellistena lichtneckerti Ermisch, 1977 . Kaszab 1979

Mordellistena micans (Germar, 1817) Kuthy 1900 
Mordellistena mihoki Ermisch, 1977 - Kaszab 1979

Mordellistena minutula Ermisch, 1956 - Ka(xab 1979

Mordellistena pseudonna Ermisch, $1956-\mathrm{Ka}$ s.ab 1979

Mordellistena reichei Emery, 1876- Kas/ab 1979

\section{Zopheridae}

Pjonomenus terebrans (O)ivier, 179()) - Merkl 1998

Colydium filiforme Fabricius, 1792 - Merkl \& Slipinski I993

Aulonium trisulcum (Geoffioy, 1785) - Merkl 1998

Synchita humeralis (Fabricius, 1792) - Coll: Sralóki

Synchita mediolanensis Villa, 1836 - Merkl 1998

Synchita separanda (Retter, I881) - Merkl \& Slipinski I99\%3

Cicones undatus Guérin-Méneville, 1829 .

Merkl \& Slipinski 1993

Bitoma crenata (Fabricius, 1775) - Merkl 1998.

Colobicus hirtus (Rossi, I790) - Merkl 1998

\section{Tenebrionidae}

Lagria atripes Mulsant \& Guillcbeau, 1855 Merkl 1998

Lagria hista (Limnacus, 1758) - Szalóki 1997, Merkl 1998

Blaps lethifera Marsham, 1802 - Merkl 1998

Gonocephalum pusillom (Fabricius, 1791) -

Coll: Szalóki

Opatrum riparium L.G.Scriba, 1796 - Merkl 1998

Opatrum sabulosum (Linnacus, 1761) -

Merkl 1998

Melanimon tibiale (Fabricius, 1781) - Coll: Szalóki

Crjpticus quisquilius (Linnacus, 1761) -

Merkl 1998

Bolitophagus intermutus Illiger, I800) - Merkl 1998 Bolitophagus reticulatus (Linnacus, 1767) -

Polinszkv 1886, Merkl 1998

Eledona agricola (Herbst, 1783) - Merkl 1998

Diaperis boleti (Linnacus, 1758) - Merkl 1998

Oplocephala haemorrhoidalis (Fabricius,

1787 ) - Polinszky 1886, Kuthy 1900

Scaphidema metallicum (Fabricius, 1792) -

Merkl 1998

Metaclisa azurea (Waltl, I 838) - Kuthy

1900, Kašab 1957

Platydema piolaceum (Fabricius, 1790) -

Merkl 1998

Siakóki

Alphitophagus bifasciatus (Say, 1823) - Coll:

Mymechixenus vaporariorum GuérinMéncville, 1843 - Merkl és Slipinski 1993
Corticeus fraxini (Kugelan, 1794) - Merkl 1998

Corticeus longulus (Gyllenhal, 1827)

(=Hypophloeus longulus) - Horvatovich 1979, Merkl 1998

Corticeus rufulus (Rosenhauer, I846)

(=Hypophloens nufulus)- Kaszab 1957

Corticeus unicolor Piller \& Mitterpacher, 1783 - Mcrkl 1998

Palonts depressus (Fabricius, 1790) - Merkl 1998 - Kuthy 1900

Tribolium madens (Charpentier, 1825) Kuthy 1900

Alphitobius diaperinus (Panzer, 1797) Merkl 1998

Diaclina fagi (Panzer, 1799) - Merkl 1998

Uloma culinaris (Linnacus, 1758) -

Horvatovich I981, Merkl 1998

Uloma rufa (Piller \& Mitterpacher, 1783) -

Horvatovich 1976, 1979, 1980, Merkl 1998

Menephilus cylindricus (Herbst, 1784) -

Horvatovich 1976, 1979, Merkl 1998

Tenebrio molitor Linnaeus, 1758 - Coll:

Szalóki

Tenebrio obscurus Fabricius, 1792 - Coll:

Szalóki

Tenebrio opacus Duftschmid, 1812 - Kaszab 1957

Cryphaeus cornutus (W.Fischer, 1823)

(=Anthracias cornutus)- Polinszky 1886

Laena viennensis (Surm, I807) - Merkl 1998

Enoplopus dentipes (Rossi, 1790) (=Enoplopus velikensis (Piller \& Mitterpacher, I783))- Kaszab 1957 1998

Stenomax aeneus (Scopoli, 1763) - Merkl

Nalassus dermestoides (Illiger, 1798) - Merkl 1998

Prionychus ater (Fabricius, 1775) (=Eryx ater)- Polinszky 1886 , Merkl 1998

Prionychus melanarius (Germar, 1813) -

Merkl 1998

Hymenalia nifipes (Fabricius, 1792) - Merkl 1998

Pseudocistela ceramboides (Linnacus, 1758) -

Merkl 1998

Gonodera lupenus (Herbst, I783) - Merkl 1998 Isomiva antennata (lanzer, 1798) - Merkl 1998 Isomira icteropa (Küster, 1852) - Kaszab 1957 Isomira murina (Linnaeus, 1758) - Merkl 1998 Isomira testacea Seidlitz, 1896 - Kaszab 1957 Mycetochara humeralis (Fabricius, 1787) -

Coll: Szalóki

Mycetochara pygmaea Redtenbacher, 1874 Kaszab 1957

Mycetochara quadrimaculata (Latreille, 1804) Merkl 1998 
Mycetocham subali Maran, 1935 - Kașzab 1957

Cteniopus sulphureus (Linnaeus, 1758) (=Cteniopus flasus (Scopoli, 1763)) - Coll: Szalóki

Omophlus rugosicollis (Brulle, 1832) - Coll: Srajóki

\section{Oedemeridae}

Sparedrus testaceus (Andersch, 1797) - Tóth 1981

Nacerdes cantiolica (Gistel, 1832) - Coll: Sralóki

Anogcodes ruficollis (Fabricius, 1781) ( $=$ Nacerdes ruficollis) - Tóth 1981

Anogcodes rufiventris (Scopoli, 1763) (=Nacerdes rufinentris)- Kaszab 1956, Tóth 1981

Anogcodes ustulata (Fabricius, 1787) (=Nacerdes ustulata)- Tóth 1981, Merkl 1998

Chrysanthia nigricornis Westerhauser, 1881 . Tóth 1981, Merkl 1998

Chrysanthia viridissima (Limnacus, 1758). Merkl 1998

Ischnomera cyanea (Fabricius, 1787) - Tóth 1981 (tévesen határozva Ischnomera caenilea (Linnacus, 1758) név alatu)

Ischnomera sanguinicolis (Fabricius, 1787) Kaszab 1956, Tóth 1981

Oedemera croceicollis (Gvllenhal, 1827) -

I'óth 1981, Merkl 1998

Oedemera femorata (Scopoli, 1763) - Tóth 1981, Merkl 1998

Oedemera flavipes (Fabricius, 1792) - T'óth 1981, Merkl 1998

Oedemera lurida (Marsham, 1802) - Tóth 1981, Merkl 1998

Oedemera podagrariae (Linnaeus, 1767). T'óth I98I, Merkl 1998

Oedemera pirescens (Linnaeus, 1767) - 'Tóth 1981, MerkJ 1998

Meloidae

Cerocoma adamovichiana (Piller \&

Mitterpacher, 1783) - Tóth 1973

Cerocoma muehlfeldi Gyllenhal, 1817 - Tóth 1973

Cerocoma schreberi Fabricius, 1781 - Tóth 1973

Epicauta rufidorsum (Goeze, 1777) - Tóth 1973

Lydus trimaculatus (Fabricius, 1787) -

Polinszky 1886

Alosimus syriacus austriacus (Schrank, 1781) -

'Tóth 1973

Oenas crassicontis (Illiger, 1800) - Tooth 1973 Lytta vesicatoria (Linnaeus, 1758) - Tóth 1973 Mylabris pannonica Kaszab, 1956 - Tóth 1973 Mylabris tenera Germar, 1834 - Tóth 1973 Mylabris variabilis (Pallas, 1782) - Tóth 1973
Meloe autumnalis Manuel, 1792 - Tóth 1973

Meloe cicatricosus Leach, 1811 - Tóth 1973

Meloe hungarzs Schrank, 1776 - Tóth 1973

Meloe mediterraneus J. Müller, 1926 - Tóth 1973

Meloe proscarabaeus Linnacus, 1758 - Tóth 1973

Meloe rufinentris Germar, 1817 (=Meloe coriarius Brandi \& Erichson, 1832)) - Tóth 1973

Meloe nugosus Marsham, 1802 - Tóth 1973

Meloe scabriusculus Brandt \& Erichson, 1832 - Tóth 1973

Meloe uralensis Paallas, 1777 - Tóth 1973

Meloe variegatus Donovan, 1776 - Tóth 1973

Meloe violaceus Marsham, 1802 - Tóth 1973, Merkl 1998

Zonitis nana Ragusa, 1882 - Tóth 1973

Zonitis praeusta Fabricius, 1792 - Polinszky 1886, Tóth 1973

Euzonitis fulvipennis (Fabricius, 1792) - Tóth 1973 Euzonitis quadrimaculata (Pallas, 1782) Tóth 1973

Euzonitis sermaculata (Olivier, 1790) - Tóth 1973

Apalus bimaculatus (Linnacus, 1761) -

Merkl 1998

Apalus bipunctatus Germar, 1817 - Tóth 1973

Sitaris muralis (Forster, 1771) - Tóth 1973

\section{Mycteridae}

Mycterus tibialis Küstcr, 1850 - Tóth 1981

Mycterus curculioides (Fabricius, I78I) - Kaszab 1956, Tóth 1981

Pythidae

Pytho depressus (Linnaeus, 1767) - Coll: Szalóki

\section{Pyrochroidae}

Pyrochroa coccinea (Limuaeus, 1761) - Tóth 1981 Pyrochroa serraticomis (Scopoli, 1763) -

'Tóth 1981, Merkl 1998

Schizotus pectinicornis (Linnaeus, 1758) Merkl 1998

Salpingidae

Lissodema cursor (Gyllenhal, 1813) - Coll: Szalóki

Lissodema denticolle (Gyllenhal, 1813) (=Lissodema quadripustulatum (Marsham, 1802)) Tóth 1981

Sphariestes castaneus (Panzer, 1796) (=Salpingus castaneus)- Tóth 1981

Vincenzellus ruficollis (Panzer, 1794) - T'óth 1981, Merkl 1998

Salpingus aeneus (Olivier, 1897) (= Rhinosimus aeneus)- Tóth 1981

Salpingus planirostris (Fabricius, 1787) (=Rhinosimus planirostris)- Tóth 1981, Mcrkl 1998 
Salpingus ruficollis (Linnacus, 1761) (=Rhinosimus ruficollis)- Merkl 1998

\section{Anthicidae}

Notoxus appendicinus (1)esbrochers des Lodges, 1875) - Tóth 1981, Merkl 1998

Notoxus miles Schmidt, 1842 - Kaszab 1956, 'lóth 1981

Notoxus monoceros (Limnacus, 1761) - Tóth 1981, Merkl 1998

Notoxus trifasciatus Rossi, 1794 - Tóth 1981 Mecynotarsus serriconis (Panzer, 1796) Tóth 1981, Horvatovich 1981, Merkl 1998

Fornicomus pedestris (Rossi, 1790) - Tóth 1981, Merkl 1998

Anthicus ater (Panzer, 1796) - Kašab 1956.

\section{Tóth $198 \mathrm{I}$}

Anthicis antherinus (Linnacus, 176I - Tóth 1981

Anthicus axillaris Schmidt, 1842 - Tóth 1981

Anthicus bimaculatus (Illiger, I 802) - Kašab

1956, Tóth 1981

Anthicus flapipes (Panzer, 1797) - Tóth 1981

Anthicus schmidti Rosenhater, 1847 - Tóth 1981

Cyclodinus humilis (Gormar, 1824)

(=Anthicus humilis)- Tóth 1981

Omonadus bifasciatus (Rossi, 1794)

(=Anthicus bifasciatus)- T'óth 1981

Omonadus floralis (Linnaeus, 1758)

(=Anthicus floralis) - Ióth 1981, MerkJ 1998

Omonadus formicarius (Gocze, 1777)
(=Anthicus formicarius)- Tóth 1981

Cordicomus gracilis (lanzer, I797)(=Anthicus gracilis)- Tóth 1981, Merkl 1998

Hirticomus hispidus (Rossi, 1792) (=Anthicus hispidus)- Tóth 1981

Hirticomus quadriguttatus (Rossi, 1792) (=Anthicus quadriguttatus)- Kaszab I956, 'lóth I981

Microhoria caliginosus pannonicus (Kaszab, 1956) (=Anthicus caliginosus pannonicus) Kaszab 1956, Tóth 1981

Endomia tenuicollis (Rossi, 1790) - Tóth 1981

\section{Aderidae}

Vanonus pruinosus (Kiesenwetter, I861) (=Aderus pruinosus)- Tóth 1981

Aderts populneus (Creutzer, 1796) - Tóth 1981

Anidonus nigtinus (Germar, 1831) (=Aderus nigrinus)- Kašab 1956, Tóth 1981

\section{Scraptiidae}

Scraptia dubia Olivier, 1790 - T'óth 1981

Scraptia fermginea Kiesenwetter, 1861 - Kaszab 1956, Tóth I981

Scraptia fuscula Müller, 182I - Tóth 1981

Cyrtanaspis phalerata (Cormar, 1831) - Kaszab 1979

Anaspis costai Emery, 1876 - Kaszab 1979 Anaspis kiesenvetteri Emery, 1876 - Kaszab 1979 Anaspis viennensis Schilsky, 1895 - Coll: Szalóki

I. táblázat: Somogy faunájából ismert fajok családsorozatonként és családonkénti megoszlása

\begin{tabular}{|l|c|l|c|}
\hline Családsorozat, Család & Fajszám & Családsorozat, Család & Fajszám \\
\hline ELATEROIDEA & 59 & Ciidae & 9 \\
\hline Drilidae & 1 & Melandryidae & 8 \\
\hline Omalisidae & 1 & Mordellidae & 16 \\
\hline Lycidae & 3 & Zopheridae & 9 \\
\hline Lampyridae & 3 & Tenebrionidae & 52 \\
\hline Cantharidae & 51 & Oedemeridae & 15 \\
\hline LXMEXYLOIDEA & 1 & Meloidae & 30 \\
\hline Lymexylidae & 1 & Mycteridae & 2 \\
\hline CLEROIDEA & 68 & Pythidae & 1 \\
\hline Trogossitidae & 2 & Pyrochroidae & 3 \\
\hline Cleridae & 16 & Salpingidae & 7 \\
\hline Melyridae & 50 & Anthicidae & 21 \\
\hline TENEBRIONOIDEA & 188 & Aderidae & 3 \\
\hline Mycetophagidae & 5 & Scraptidae & 7 \\
\hline
\end{tabular}




\section{Irodalom}

Horvntovich, S. 1969: A Kárpátmedencei lágytestü bogarak (Col., Malacodermata) faunisztikai és fenológiai adatai. - Folia ent. hung. 22: 131-249.

Horvntovich, S. 1976: Ritka bogárfajok a Barcsi Ösborókás és a Villányi-hegység területérôl - Dunántúli Dolgozatok Természettudományi Sorozat 10: 47-49.

Horvatovich, S. 1979: Hazánk faunájára új és ritka bogárfajok a Dél- és Nyugat-Dunántúlról (Coleoptera). - $\Lambda$ Janus Pannonius Múzeum Évkönyve (1978), 23: 31-39.

HorvNTOVICH, S. 1980: Hazánk faunájára új és ritka bogárfajok a Dél- és Nyugat-Dunántúlról, ;II. (Colcoptera). - A Janus Pannonius Múzeum Évkönyve (1979), 24: 33-43.

HorvNiovich, S. 1981 : Hazánk faunájára új és ritka bogárfajok a Dél-és Nyugat-Dunántúlról, III. (Coleoptera). - A Janus Pannonius Múzeum Évkönyve (1980), 25: 71-83.

KuszıB, Z. 1955: Lágytestú bogarak, Malacodermata - In: Magyarország Állatvilága (Fauna Hungariac), VIIII, 1. Akadémia Kiadó, Budapest, 144 pp.

Kısz^в, Z. 1956: Felemás lábfejízes bogarak III., Heteromera III. - In: Magyarország Állatvilága (Fauna Hungariac), IX, 3. Akadémia Kiadó, Budapest, 108 pp.

KıszAв, Z. 1957: Felemás lábfejízes bogarak I., Heteromera I. - In: Magyarország Állatvilága (Fauna Hungariac), IX, 1. Akadémia Kiadó, Budapest, 126 pp.

Kuszıв, Z. 1979: Felemás lábfejízes bogarak II., Heteromera II. - In: Magyarország Állatvilága (Fauna Hungariae), IX, I. Akadémia Kiadó, Budapest, I00 pp.

KuThy, D. 1900): Coleoptera. - In: Fauna Regni Hungariac, III. Arthropoda, 5-214. pp. - K. M. Term.tud. Társulat, Budapest

LAwrence, J. F. \& Newton Jr. A. F. 1995: Coleoptera. Families and subfamilies of Coleoptera (with selected genera, notes, references and data on family-group names) - In: Pakulak, J and Slipinski, S. A. (eds.): Biology, Phylogeny, and Classification of Coleoptera: Papers Celebrating the 80th Birthday of Roy $\Lambda$. Crowson., Muzeum i Instytut Zoologii PAN, Warszawa, 779-1006.

Lohse, G. A. \& Lucht W. H. (1992): Die Käfer Mitteleuropas, 2. Supplementband mit Katalogteil, Goecke \& Evers, Krefeld, 375 pp.

MerkL, O. \& SLIPINSKI, S. A. (1993): Különbözö csápú bogarak VI. - Diversicornia VI., Bunkóscsápú bogarak VIII. - Clavicornia VIII. - In: Magyarország Állatvilága (Fauna Hungariac), VIII, 16., Akadémia Kiadó, Budapest, 75 pp.

MERKL, O. 1993: Különbözố csápú bogarak VI. - Diversicornia VI., - Bunkóscsápú bogarak I. Clavicornia I. - In: Magyarország Állatvilága (Fauna Hungariae), VIII, 8., Akadémia Kiadó, Budapest, 26 pp.

MerkL, O. 1998: Data to 46 beetle families (Coleoptera) from the Duna-Dráva National Park, South Hungary - In: Uherkovich , Á. (ed.): A Dráva mente állatvilága II. Dunántúli Dolgozatok Természettudományi Sorozat 9: 209-232

Polinszky, E. 1886: Adatok Somogymegye bogárfaunájához - Rovartani Lapok 3: 146-148

SÁr, J. Dudós, Cì. \& Merke, (). 200) l: ^ lapos sárkánybogár (Pytho depressus) elsó bizonyítout magyaroršági clőfordulása a Villányi- hegységben (Coleoptera: Pythidac). - Természetvédelmi Közlemények 9: (in print)

Slipinski, S. A. \& LAWRENCE, J. F. 1999: Phylogeny and classification of Zopheridae sensu novo (Coleoptera: Tenebrionoidea) with a reviev of the genera of Zopherinae (excluding Monommatini). - Annales Zoologici 49 (1-2): 1-53.

SzALóKı, D. 1997: A Mátra Múzeum bogárgyújteménye, Malacodermata és Heteromera (részben) (Coleoptera). - Folia Historico Naturalia Musci Matraensis, 22: 145-174.

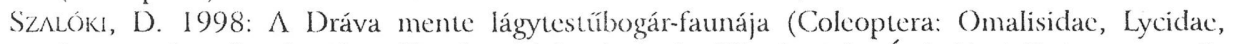
Lampyridac, Cantharidae, Cleridae, Melyridae) - In: Uherkovich , Á. (ed.): A Dráva mente állatvilága II. Dunántúli Dolgozatok Természettudományi Sorozat 9: 233-236

Tóth, L. 1973: A Kárpátmedence feleméslébfejízes bogarainak (Col., Heteromera) lelóhelyadatai 1. - Folia cnt. hung. 26. 1: 165-192.

Tó̀t, L. 1981: Fundortangaben der Heteromeren (Coleoptera: Heteromera) aus dem KarpathenBecken. - Folia ent. hung. 42(34). 2: 221-237.: 


\section{Catalogue of the Malacodermata an Heteromera of Somogy county (Coleoptera: Elateroidea (in part), Lymexyloidea, Cleroidea, Tenebrionoidea)}

\section{DEZSÖ SZALÓKI}

In the present paper, author gives the names of 316 beetle species ( 59 Elateroidea (in part), I Lymexyloidea, 68 Cleroidea, 188 Tenebrionoidea) originated from Somogy county, which forms the $53 \%$ of the whole Hungarian fauna. The first occurrence of Pytho depressus (Linnaeus, 1767) in Hungary is known from this region. The exact locality of this species is recorded at Pusztakovácsi, Alsókölked, 18.X.1997., leg.: Gy. Rozner. Several specimens of this species were collected later by Gy. Dudás, S. Horvatovich and J. Sár in the Villányi hills. (Sর́k et al 200I).

Table I summarises the species gathered from Somogy county listed according to families and superfamilies.

The enumeration of species is compiled by the works of P(OLINSZKY (1886), Kuthy (1900), Kaszab (1955, 1956, 1957, 1979), Horvatovich (1969, 1976, 1979, 1980, 1981), TóTH (1973, 1981), MERkL \& SLIPINSKI (1993), Merkl (1993, 1998), SÁr et al (2001), SzALÓKI (1997, 1998) and by the collection of Szalóki.

Ischnomera caerulea (Linnaeus, 1758) (Oedemeridae) was misidentified by TóTH (1981), since all the specimens from Somogy county housed in the collection of the Hungarian Natural History Museum were proved to be Ischnomera cyanea (Fabricius, 1787). The error is due to the fact that Ischnomera cyanea was treated by KASZAB (1956) as synonym name of I. caerulea. On the labels of older specimen figures both the names cyanea and caerulea can be found. The extremely similar two species clearly differ from each other in the shape end of paramera and penis (LOHSE \& LUCHI (1992)).

The southern coast of Lake Balaton changed dramatically in the last decades, it was built up, in some places drained, therefore the former rich fauna of the region of Siófok, Zamárdi and Balatonöszöd is strongly transformed. Obviously, the reduction of the late expended steppe region led up to the decrease of number of specimens and species of the family Meloidae.

Several names used in the literature differ from those considered valid today; in these cases the original name is found in brackets and with the mark of equation $(=)$ after the name considered valid today.

Author's address:

Dezsố SZALOKKI

Radnóti Miklós Teacher Training School

H-1146 Budapest

Cházár András utca 10.

HUNGARY

e-mail:meloe@server.radnoti-elte.sulinet.hu

or

H 1118 Budapest

Villányi út 113.

HUNGARY 